Conclusions These results demonstrated that lycopene protects against I/R-injury in vitro, which may be attributable to its roles in preventing ROS-mediated apoptosis.

\section{GW23-e0577 LYCOPENE PROTECTS CARDIOMYOCYTES AGAINST ISCHAEMIA/REPERFUSION-INJURY BY PREVENTING APOPTOSIS}

doi:10.1136/heartjnl-2012-302920a.168

Rongchuan Yue, Houxiang Hu, Tao Luo, Ke Li, Lei Xu, Shuang Zhang, Houxiang Hu. Department of Cardiology, Affiliated Hospital of North Sichuan Medical College, Nanchong, Sichuan, China, 637000

Objectives Reactive oxygen species (ROS)-mediated calpain activation has shown to play an important role in cardiomyocyte apoptosis. Lycopene is a natural antioxidant carotenoid that has been shown to have protective properties on cardiovascular system. However, whether lycopene can protect cardiomyocytes from ischaemia/reperfusion (I/R) injury, and the mechanisms of lycopene's effects are not clear.

Aim The purpose of this study was to investigate whether lycopene could efficiently protect against I/R-injury, and to elucidate the possible mechanism of its actions.

Methods Cultured cardiomyocytes from neonatal C57BL/6 mice were exposed to $4 \mathrm{~h}$ hypoxia followed by $8 \mathrm{~h}$ reoxygenation to simulate I/R-injury. Cardiomyocytes were divided into three groups: control, I/R, I/R+lycopene group ( $0.5 \mu \mathrm{M}$ lycopene pretreated for $4 \mathrm{~h}$ before $\mathrm{I} / \mathrm{R})$. The apoptosis index of cardiomyocytes was counted by comparing TUNEL-positive counts with the total cell nuclei determined by Hoechst 33342 counterstaining. The intracellular ROS levels were quantified by determination of intracellular oxidant production based on the oxidation of $2^{\prime}, 7^{\prime}$-dichlorofluorescin diacetate (DCFH-DA), and the activity of caspase-3 was also determined in these groups.

Results The apoptosis index was significantly increased in I/R group compare to control, and decreased in $\mathrm{I} / \mathrm{R}+$ lycopene group (10.37 $\pm 1.25 \%, 32.03 \pm 4.79 \%$ and $22.57 \pm 3.22 \%$, respectively). The intracellular ROS level in I/R group was higher compared to control and lower in $\mathrm{I} / \mathrm{R}+$ lycopene group $(1.05 \pm 0.12,2.40 \pm 0.27$ and $1.88 \pm 0.18$, respectively). The caspase-3 activity in $\mathrm{I} / \mathrm{R}$ group was significantly increased compared to control and decreased in $\mathrm{I} / \mathrm{R}+$ lycopene group $(1.02 \pm 0.19,2.27 \pm 0.64$ and $1.42 \pm 0.19$, respectively). 